Referencia para citar este artículo: Marín-Díaz, V. \& Sánchez-Cuenca, C. (2015). Formación en valores y cuentos tradicionales en la etapa de educación infantil. Revista Latinoamericana de Ciencias Sociales, Niñez y Juventud, 13 (2), pp. 1093-1106.

\title{
Formación en valores y cuentos tradicionales en la etapa de educación infantil ${ }^{*}$
}

\author{
VERÓNICA MARÍN-DÍAZ ${ }^{* *}$ \\ Profesora Universidad de Córdoba, España. \\ CARMEN SÁNCHEZ-CUENCA ${ }^{* * *}$ \\ Profesora Colegio Miguel Ángel Ortiz Belonte, España.
}

Articulo recibido en marzo 26 de 2014; artículo aceptado en mayo 19 de 2014 (Eds.)

- Resumen (analítico): Los valores son hoy un elemento de la formación, siendo pieza clave en la Educación Infantil. Junto a estos encontramos la metodología de los cuentos tradicionales como forma de enseñanza-aprendizaje. En el presente artículo exponemos los primeros resultados de un estudio no experimental, llevado a cabo con el objetivo general de conocer cuáles son los valores que el profesorado cree que transmiten los cuentos infantiles. El principal resultado obtenido es que los cuentos tradicionales transmiten diferentes valores en función de la etapa educativa en que se empleen y de la experiencia profesional de los sujetos docentes. Concluimos que la educación en valores a través de los cuentos tradicionales en la etapa de Educación Infantil, es una metodología válida para la socialización de los niños y las niñas.

Palabras clave: infancia, investigación curricular, educación en la primera infancia, aprendizaje, investigación educativa (Tesauro de Ciencias Sociales de la Unesco).

Palabras clave autoras: cuentos, valores.

\section{Values education and traditional stories in early childhood education}

- Abstract (analytical): Nowadays values are an important element of education and represent a key component of Early Childhood Education. In addition, traditional stories are used in teaching and learning methodologies. This article describes the initial results of a non-experimental study that had the general objective of identifying the values that teachers believe are transmitted by traditional stories for children. The main result obtained was that traditional stories and fairytales transmit different values depending on the educational stage of the students and the professional experience of teachers. The study concludes that values education through traditional stories in early childhood education is a valid methodology to facilitate the socialization of children.

Key words: childhood, curriculum research, early childhood education, learning, educational research (Unesco Social Sciences Thesaurus).

Authors key words: tales, values.

\footnotetext{
Este artículo corto se deriva del trabajo que las autoras estamos realizando dentro del marco del proyecto de tesis doctoral denominado "Estudio y desarrollo de valores a través de los cuentos mediante metodologías tradicionales Vs. TIC, en la etapa de Educación Infantil”, dentro de la escuela de doctorado de Ciencias Sociales y Jurídicas de la Universidad de Córdoba (España), código 532-Programa de Doctorado en Ciencias Sociales y Jurídicas. Fecha de inicio septiembre de 2012 y fecha de finalización, diciembre de 2016. Área de conocimiento: Ciencias de la Educación, Subárea de conocimiento: Educación general.

** Dra. en Pedagogía por la Universidad de Granada (España), profesora Titular de la Universidad de Córdoba (España). Correo electrónico: vmarin@uco.es

*** Maestra especialista en Educación Infantil. Profesora de Educación Infantil del centro "Miguel Ángel Ortiz Belmonte". Doctoranda de la Universidad de Córdoba (España). Correo electrónico: casacu32@hotmail.com
} 


\section{Formação em valores e histórias tradicionais no palco da educação infantil}

- Resumo (analítico): Os valores são hoje um elemento na formação, sendo fundamental na Educação Infantil. Junto com isso, encontramos a metodologia de histórias tradicionais, como forma de ensino e aprendizagem. Neste artigo, apresentamos os primeiros resultados de um estudo não-experimental realizado com o objetivo geral de conhecer quais os valores que os professores acreditam que são transmitidos nas histórias infantis. O principal resultado é que os contos tradicionais transmitem valores diferentes dependendo do estágio educacional empregado e a experiência profissional dos professores. Conclui-se que a educação de valores através de contos tradicionais é válida para a socialização das crianças.

Palavras-chave: infância, investigação currículo, educação infantil, aprendizagem, pesquisa educacional (Thesaurus de Ciências Sociales da Unesco).

Palavras-chave autores: histórias, valores.

-1. Introducción. -2. La educación infantil hoy. -3. Los valores en la educación infantil. -4. Los cuentos infantiles y su valor educativo para el desarrollo de actitudes. $\mathbf{- 5}$. Método. $\mathbf{- 6 .}$ Resultados. -7. Conclusiones. -Lista de referencias.

\section{Introducción}

¿Transmiten valores los cuentos infantiles? Esta es una de las preguntas clave que la enseñanza en valores trabaja desde la más temprana edad. La intencionalidad de esta acción es que exista una continuidad que permita ir desarrollando y depurando las actitudes del niño o niña frente a cada valor. Otra cuestión fundamental en torno a este tema es ¿qué valores transmiten los diversos y variados cuentos que se trabajan en esta etapa? Una de las principales respuestas que aportan los individuos profesionales de la educación es que, según la temática del cuento y los personajes que intervienen, se van a potenciar más unos valores u otros.

Hasta ahora se ha considerado que la educación en valores tiene una gran relevancia en esta etapa, pues conjuga un enfoque globalizado del mundo que los niños y niñas en ese momento tienen de la vida que les rodea (Amei-Waece -Asociación Mundial de Educadores Infantiles-, 2010), con todos aquellos aspectos que rodean e inciden en su proceso de socialización. La construcción de ese mundo se apoya en una perspectiva que haga posible la aceptación, por la sociedad en general, de que todos los individuos son iguales, tal y como los niños y las niñas lo conciben y entienden (García \& Gallego, 2011). Dicha visión debe comenzar su andadura apoyándose en una perspectiva que suponga la confrontación de los valores y los contravalores que la sociedad ha admitido como generales y válidos para todas y todos (Marín-Díaz, Llorent, González \& Mata, 2005). Todo ello tiene que ser hoy en día uno de los objetivos principales de la educación en la etapa infantil, debido -principalmente- a que cada vez se hace más complejo fomentar este tipo de elementos, y la cultura de la impersonalidad está llegando a cotas extremas. En este trabajo, tratamos de establecer cuáles son los valores que transmiten los cuentos infantiles empleados por el profesorado de Educación Infantil como recurso didácticocurricular y que posibilitan el desarrollo de estos. Partimos de la conceptualización que del término usa Paya (1997, p. 156) al entenderlo como la forma de "integrar a las personas en el conjunto de normas, valores y pautas de acción de la sociedad a la que pertenece", de ahí que compartimos con Echevarría (2003), Pestaña (2004) y Amei-Waece -Asociación Mundial de Educadores Infantiles- (2010), que este debe ser un elemento transversal del currículo de esta etapa, y de ahí surge nuestro interés en esta investigación.

El objetivo general que buscamos pasa por recoger la opinión de un grupo de docentes que trabaja en la etapa de Educación Infantil, en torno al empleo de los cuentos infantiles como medio para potenciar el desarrollo de una educación en valores, y así poder generar conclusiones 
de acción aplicables a los diferentes grupos de clase.

\section{La educación infantil hoy}

Tradicionalmente se ha venido presentando el desarrollo socio-afectivo, como un cuerpo de estudios unitario en el que se insistía en la mutua interdependencia entre las distintas facetas que implican crecimiento social, crecimiento afectivo y desarrollo de la personalidad. Es difícil que puedan darse teorías o intentos de explicación de estos desarrollos por separado, al igual que arduamente se puede explicar el desarrollo cognitivo sin entender el físico o psicomotor, o la influencia que el desarrollo socio-afectivo tiene sobre la construcción de las estructuras cognitivas de cualquier individuo.

En líneas generales, podemos partir de dos premisas que, a nuestro juicio, marcan el desarrollo socio-afectivo del niño o niña de esta etapa: de un lado, la existencia de múltiples contextos significativos. Se trata de una construcción que se realiza en diferentes ambientes: familiar, escolar, la calle, etc., y que se relacionan entre sí. Por otro lado, nos encontramos con una doble finalidad; esto es, conseguir la integración social del niño o niña, al mismo tiempo que se potencia su diferenciación como individuo autónomo, con espacios propios en el marco social y con capacidad de actuar y modificar a la vez el propio entorno social.

En lo que se refiere al desarrollo y al aprendizaje, según la perspectiva que ha aportado Vigotsky (1984) y desde la visión de Bassedas, Huguet y Solé (1998), el aprendizaje escolar ha sido fundamental para poder razonar y entender cuál es la naturaleza de este y del proceso educativo, además de tratar de determinar las relaciones de todo ello con el crecimiento del individuo. A la vez, el aprendizaje escolar facilita y promueve el desarrollo mediante la creación de zonas de desarrollo, potencia que, según lo mencionado, puede definirse como

(...) la distancia entre el nivel actual de desarrollo, determinado por la capacidad de resolver independientemente un problema, y el nivel de desarrollo potencia, determinado a través de la resolución de un problema con la orientación de un adulto o la colaboración de un compañero más capaz (Vigotsky, citado por Riviere, 1984, p. 98).

Y por último, no podemos olvidar el papel del profesorado en el proceso de formación de los niños y niñas. ¿Qué capacidades desarrollan los sujetos docentes en el niño o niña como respuesta a su propia conducta? El alumnado de Educación Infantil debe ser considerado como un todo; la escuela debe encargarse -conjuntamente con la familia- del desarrollo integral de su personalidad. La escuela, en primera instancia, debe constituirse como un espacio de formación y socialización, donde el niño o niña pueda aprender a vivir, creciendo no solo en su formación académica sino también en la moral y ética (Echevarría, 2003); es decir, formar su mente y su alma. En este proceso, un pilar básico será la familia la que ha de compartir el ideario del centro para poder crecer a los niños y niñas como sujetos activos y críticos con la sociedad. Desde esta perspectiva, la infancia debería ser considerada como una etapa que tiene importancia en sí misma, no solo por lo que vendrá después, si no por ser una edad con características propias y específicas que hay que vivir con plenitud e intensidad. La Educación Infantil ya no se entiende como potestad de las familias ni de sus circunstancias sociolaborales y económicas, sino como derecho de la persona desde su nacimiento.

\section{Los valores en la educación infantil}

Al hablar de los valores debemos puntualizar algunas cuestiones. En primer lugar, hemos de acentuar el carácter real de los mismos. Debe quedar claro que estos no son ficciones, ni objetos de la imaginación pertenecientes, por tanto, al mundo de lo fantástico o imaginario. Sino todo lo contrario: pertenecen al mundo de lo real. Son realidades enraizadas en nuestra cultura. Desde estos pensamos y actuamos, y son los que deciden y dan explicación y coherencia a nuestra vida. No están fuera de nosotros, son como el aire que respiramos, vivimos en ellos. Podemos afirmar que se encarnan en realidades concretas y se expresan a través de ellas, pero a la vez desbordan el lugar de su encarnación 
como expresión del yo de la persona (López, 2005).

Cuando se aborda el mundo de los valores es importante, más aún imprescindible, rescatar la realidad de estos, extrayéndola del mundo de la vaguedad y de la fantasía. Solo así podrá ser presentado como algo valioso, noble, algo por lo que merezca la pena esforzarse. El peligro del engaño o de la propuesta falaz acecha constantemente, acercando la falsa idea establecida sobre la realidad del propio valor. De aquí que el primer paso en la formación de los valores a través de la educación, sea el descubrimiento de los mismos como realidad operativa en la vida de toda persona. Identificarlos, ponerles nombre, vincularlos a comportamientos de personas concretas y -preferentemente- a las conductas propias, requisito indispensable en este tipo de educación, aun corriendo el riesgo de tener que asumir que, junto a una experiencia valiosa, se da también en el mismo sujeto el contravalor o la conducta reprobable, provocando que nunca se abandonen las propuestas abstractas, inoperantes e ineficaces del valor en sí mismo y de todo lo que ello conlleva para el desarrollo de la persona.

En segundo lugar, debemos resaltar su carácter inevitable. Ineludiblemente los valores se producen en tanto que la persona es un ser de valores. No se la puede entender sin la presencia de estos; la construcción del yo pasa por la "apropiación" de ellos, por la asimilación del sentimiento de propiedad y pertenencia de ellos. De aquí que sean entendidos como algo cotidiano que acontece en la vida de toda persona. Forman parte de la rutina de nuestra existencia diaria, de tal modo que constituyen "unos excelentes termómetros que marcan el grado de integración o de desintegración que experimentan los diversos grupos sociales" (Duch, 2003, p. 151).

En tercer y último lugar, hay que atender a las diversas clasificaciones que sobre ellos podemos encontrar en la literatura; así, Poma (2011) los agrupa en: morales, estéticos, sensibles, vitales, útiles, lógicos, religiosos y cívicos, mientras que Trilla (1995) plantea la clasificación más desde una perspectiva filosófica que pedagógica, a saber: Compartidos
(A) y No compartidos: Contradictorios con A (contravalores) (B) y no contradictorios con A (C), donde los A serán aquellos que son aceptados de forma generalizada, como pueden ser la bondad, el amor, la paz, el respeto mutuo, etc.; los B son aquellos que "no gozarían de una aceptación generalizada" (racismo, autoritarismo, separatismo, etc.), serían contravalores y por último los $\mathrm{C}$, "tampoco serían compartidos, pero que, en cambio, no serían percibidos de forma generalizada como contradictorios con los valores A" (Trilla, 1995, p. 103).

Por otra parte, los valores en cuanto a objetivos constituyen fines de la actividad humana; y en lo que se refiere a la motivación de la conducta, se instituyen como los principios de la misma. Y si son principio y fin de la acción humana, lo son también de la educación.

Si los valores se caracterizan por ser cualidades -se convierten en cualidades en el momento en que se refieren a un objeto- $y$ por no poder existir por sí solos (Marín-Díaz et al., 2005), será necesario que se desarrolle una formación que capacite para la consecución de los procesos de socialización por el que los sujetos pasan; de ahí que exista una vinculación entre educación y valores.

La educación, en efecto, se guía por el panel de los valores, en cuanto a ideales del proceso educativo. Educar es hacer que el educando ponga los valores en su vida, que los conozca, los estime, los aproveche y los haga crecer. La consecución de una buena educación podría medirse por la calidad y la cantidad de los valores logrados en y por el sujeto estudiante, pues de lo que se trata, en definitiva, es de que la jerarquización que este haga de ellos sea rica y variada. También se trata de que sea una escala correcta, es decir, que se conforme a la jerarquía axiológica objetiva existente, evitando las personales inversiones de estos en contravalores (Quintana, 2005).

Teniendo en cuenta la dificultad para describir y acotar todos los contenidos de la educación en valores, pasamos a enumerar los más relevantes e importantes en la educación de los niños y niñas, que a nuestro juicio son: "consolidación del respeto mutuo y la comprensión, la solidaridad, la tolerancia, la 
igualdad, la cooperación y ayuda, la generosidad, la responsabilidad, la paz, la justicia, la libertad y la verdad" (Rollano, 2004, p. 45), los cuales van a marcar la investigación que realizamos y que exponemos más adelante.

El sistema educativo español definido en la normativa aprobada recientemente (Cortes Generales de España -Ley Orgánica para la mejora educativa-, 2013), incluye entre sus finalidades proporcionar a los alumnos y alumnas una formación que favorezca los diferentes aspectos de su desarrollo, lo cual supone e incluye la construcción de un conjunto de valores que no siempre se adquieren de manera espontánea. Estos, básicamente, están referidos a los ámbitos de la convivencia y a la vida social del sujeto, encontrándose relacionados -en gran medida- con las necesidades, demandas y problemas cuya evolución reciente hace necesario su tratamiento en el centro educativo, y suponen una importante contribución a la mejora de la calidad de la enseñanza.

\section{Los cuentos infantiles y su valor educativo para el desarrollo de actitudes}

El cuento es un agente motivador que despierta gran interés en los niños y niñas, permitiéndoles convertir lo fantástico en real, identificar personajes, dar rienda suelta a su fantasía, a su imaginación y a su creatividad, además de suavizar tensiones y resolver conflictos (Martín, 2006). Al identificarse con los sujetos protagonistas de los cuentos, los niños y niñas están mejor dotados, también, para comprender sus propias dificultades $\mathrm{y}$ poder considerar confiadamente en la idea de que un día llegarán a superarlas.

Por otra parte creemos necesario, antes de adentrarnos más en el tema, determinar para qué sirven los cuentos en educación. Apoyándonos en Bruder (2000) podemos indicar que los cuentos ayudan en el desarrollo de la creatividad, de la inteligencia, de las emociones, en la estimulación del lenguaje, en la capacidad de separar la fantasía de la realidad, en aprender a dar y recibir, en el desarrollo del arte, en aprender la lectura subliminal, en jugar, en aprender a enfrentar conflictos y en desarrollar una identidad armónica, entre otros aspectos.

Un elemento principal -a nuestro juicioa la hora de emplear los cuentos en el aula infantil, es determinar cuáles serán los que en cada nivel educativo se van a utilizar. Para ello se han de determinar una serie de criterios para seleccionarlos y narrarlos tanto de forma oral como escrita. Siguiendo a Ortega y Tenorio (2006), los cuentos se pueden clasificar atendiendo a sus características en:

- Adaptarse a la etapa evolutiva del niño o niña.

- Ser breves y adaptados a su capacidad de atención.

- Ser sencillos y claros, tanto en el lenguaje empleado como en su estructura. No debe haber demasiados personajes, ya que el niño o niña puede equivocarse en la trama.

- Tener una presentación de una estructura lineal y fuertes contrastes.

- Tener notas de humor para acercarse al alumnado y captar su interés.

- Tratar de conseguir la participación a través de distintas onomatopeyas, respuestas aisladas y reiteradas además de sus comentarios.

Ahora bien, también se pueden clasificar en función de la edad o del contenido, tal y como hacen Medina (1973), Bruder (2000), o Martín (2006). Vinculando ambos aspectos apostamos por la agrupación de Teres y García (1997, pp. 69-70), quienes se centran en las edades del segundo ciclo de Educación Infantil: 3, 4 y 5 años (ver tabla 1). 
Tabla 1. Relación de cuentos por edades.

\begin{tabular}{|lll|}
\hline \multicolumn{1}{|c|}{ 3 años } & \multicolumn{1}{c|}{5 años } & \\
\hline & Blancanieves y los siete enanitos & La Bella y la Bestia \\
& Pulgarcito & La bella Durmiente \\
Garbancito & Caperucita Roja & La ratita presumida \\
El Zapatero y los Duendes & La casita de chocolate & La lechera \\
Guillermo Tell & El traje nuevo del Rey & El gato con botas \\
El enano saltarín & Los tres cerditos & Pinocho \\
Juan sin miedo & El soldadito de plomo & Peter Pan \\
Simbad el marino & El sastrecillo valiente & Los músicos de Bremen \\
Robin Hood & Aladino y la lámpara maravillosa & Los viajes de Gülliver \\
La princesa y el guisante & Ali Babá y los 40 ladrones & Lallina de los huevos de oro \\
Ratón de Campo y Ratón de & Las habichuelas mágicas & Pulgarcito \\
Ciudad & El patito feo & Los siete cabritillos \\
El flautista de Hamelín & La sirenita & El príncipe encantado \\
& La vendedora de cerillas & El príncipe feliz y la golondrina \\
& Alicia en el país de las maravillas & La reina de las Nieves \\
& La cigarra y la hormiga & Ratón de Campo y Ratón de ciudad \\
& & El ruiseñor del Emperador \\
\hline
\end{tabular}

El valor educativo del cuento procede de sí mismo y de su función pedagógica. Es un recurso que, utilizado en las aulas para la transmisión de valores, proporciona un acercamiento a la cultura en la que se socializa, satisface las necesidades cognitivo-afectivas de los niños y las niñas, pone en contacto al alumnado con un lenguaje culto y elaborado, favorece el desarrollo de la fantasía y la imaginación, y libera tensiones. Es un importante recurso globalizador, es un recurso didáctico que permite globalizar multitud de contenidos, al posibilitar variedad de actividades en torno a el (Ortega \& Tenorio, 2006).

Podemos terminar la idea llamando la atención sobre el estudio realizado por González (2006), en el que se ha comprobado el poder de los cuentos entendidos más allá de su utilización para trabajar diversos contenidos del currículo, radicando en su capacidad de transmisión de roles, creencias y valores, lo que favorece el desarrollo social-afectivo del alumnado.

\section{Método}

Teniendo en cuenta la importancia que tiene el desarrollo de los valores para la convivencia en el mundo actual, hemos desarrollado un trabajo de investigación sobre la educación en estos, considerada como un pilar de la formación y del proceso de enseñanza-aprendizaje, proceso que desde todos los niveles educativos se trabaja a través de los cuentos infantiles empleados por los docentes y las docentes como recurso de aula. En nuestro trabajo, partimos de un interrogante muy concreto: queremos establecer cuáles son los valores que transmiten los cuentos infantiles, utilizados por el profesorado de la etapa de Educación Infantil como recurso didácticocurricular. Ubicándonos dentro del espectro de la metodología cuantitativa, este estudio responde a un diseño empírico no experimental, descriptivo y correlacional, dada la naturaleza de los objetivos que hemos pretendido conseguir; no obstante, queremos resaltar que el mismo puede ser completado desde una perspectiva cualitativa que aporte la subjetividad que este tipo de estudio requiere, aspecto este que por motivos de espacio no exponemos aquí. Hemos partido de un modelo estructural relacional de las variables dimensionadas en dos aspectos que son: datos de identificación del profesorado participante y valoraciones de doble entrada, donde relacionamos los cuentos seleccionados con los valores elegidos que se pueden fomentar en esos cuentos infantiles. 
A partir de este cuestionamiento de partida, concretamos este en el presente objetivo general, que no es otro que conocer cuáles son los valores que el profesorado cree que transmiten los cuentos infantiles según la edad del alumnado. Este objetivo general lo concretamos en los siguientes objetivos específicos, los cuales se apoyan en la concepción de que un cuento puede transmitir un único valor o más de uno, entendiendo este aspecto desde una perspectiva holística alejada de cualquier halo de negatividad o subjetividad:

1. Describir los valores que transmiten los cuentos infantiles seleccionados.

2. Determinar el número de valores que puede transmitir un cuento.

3. Determinar los valores que según los profesores y profesoras adquirirá el estudiantado según el nivel educativo en que se encuentre

4. Encontrar los valores que transmiten los diferentes cuentos estudiados en función de los años de experiencia profesional.

Las hipótesis de las que partimos son:

- Los cuentos pueden transmitir más de un valor.

- Los cuentos pueden ser empleados como elemento reforzador de un valor una vez se ha adquirido.

- Los cuentos ayudan al docente en el desarrollo de la educación en valores.

Una vez determinado el propósito que rige este estudio, especificamos las variables que dan cuenta de los fenómenos a estudiar. Para su selección, hemos considerado aquellos valores que cree el profesorado que desarrollan los cuentos en cada nivel de Educación Infantil. Estos valores son muy variados, por lo que, para su definición, hemos empleado dos criterios bien diferenciados: de un lado, los datos de identificación del profesorado encuestado y de otro, y muy próximo al modelo Cipp (contexto, entrada, proceso, producto) de evaluación planteado por Stufflebeam y Shinkfield (1990), tuvimos en cuenta la función que las variables desempeñan en el contexto de estudio: variables de entrada (características del profesorado), variables del proceso (uso de cuentos infantiles en el aula) y variables de producto (los valores que transmiten los cuentos infantiles).
Parala obtención delainformación referente a estas variables recurrimos a la construcción de una escala dirigida al profesorado del nivel de Educación Infantil. Esta está conformada por una serie de ítems sobre los que se pide al sujeto que manifieste una opinión determinada; es una agrupación progresiva de valores, cada uno de los cuales constituye un valor estándar, los que son utilizados para medir diversos tipos de datos. Empleamos la escala como técnica de recogida de datos en esta investigación, pues permite dar respuesta a los objetivos de investigación planteados.

A través de este instrumento obtuvimos datos por medio de preguntas realizadas a los diferentes docentes de Educación Infantil, cuyo fin es recoger información referente al tema investigado, en este caso las opiniones que tiene el profesorado respecto al desarrollo de valores a través del trabajo con los cuentos infantiles.

Dicha escala consta de dos partes: en la primera se recogen datos de tipo descriptivo: sexo, edad, tipo de centro, titulación, nivel educativo que imparte y años de experiencia docente. $\mathrm{Y}$ en la segunda parte se presenta una tabla de doble entrada (ver tabla 2), construida a partir de a) las investigaciones realizada por Teres y García (1997) sobre los cuentos que se trabajan en Educación Infantil, distribuidos por edades (columna vertical), y b) las investigaciones realizadas por Rollano (2004) donde, siguiendo su teoría, se establecen los valores que son más importantes en la educación del alumnado de estas edades (columna horizontal). Se trata, pues, de que el profesorado al que se le ha administrado este instrumento vaya relacionando con una cruz el valor que considera que se trabaja de entre todos los señalados, en cada uno de los cuentos referidos. 
Tabla 2. Escala de valoración de cuentos y valores.

\begin{tabular}{|c|c|c|c|c|c|c|c|c|c|c|c|c|}
\hline & 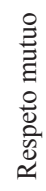 & 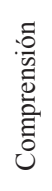 & 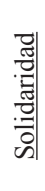 & 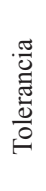 & 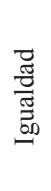 & 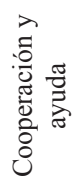 & $\begin{array}{l}\overrightarrow{0} \\
\frac{0}{0} \\
0 \\
0 \\
0 \\
0 \\
0 \\
0\end{array}$ & 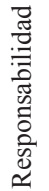 & ฮี & 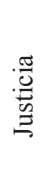 & 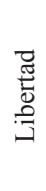 & \\
\hline \multicolumn{13}{|l|}{ El patito feo } \\
\hline \multicolumn{13}{|l|}{ Caperucita Roja } \\
\hline \multicolumn{13}{|l|}{ El flautista de Hamelín } \\
\hline \multicolumn{13}{|l|}{ Hansel y Gretel } \\
\hline \multicolumn{13}{|l|}{ La Bella Durmiente } \\
\hline \multicolumn{13}{|l|}{ El gato con botas } \\
\hline \multicolumn{13}{|l|}{ Los tres cerditos } \\
\hline \multicolumn{13}{|l|}{ Peter Pan } \\
\hline \multicolumn{13}{|l|}{ La Cenicienta } \\
\hline \multicolumn{13}{|l|}{ Los 7 cabritillos } \\
\hline \multicolumn{13}{|l|}{ Los tres osos } \\
\hline \multicolumn{13}{|l|}{ Pulgarcito } \\
\hline \multicolumn{13}{|l|}{ Pinocho } \\
\hline \multicolumn{13}{|l|}{ Blancanieves } \\
\hline \multicolumn{13}{|l|}{ Bambi } \\
\hline \multicolumn{13}{|l|}{ La lechera } \\
\hline \multicolumn{13}{|l|}{ El mago de Oz } \\
\hline \multicolumn{13}{|l|}{ La Bella y la Bestia } \\
\hline El libro de la selva & & & & & & & & & & & & \\
\hline
\end{tabular}

Fuente: Elaboración propia.

Para la validación de contenido del instrumento, lo sometimos al juicio de 5 individuos expertos, docentes especialistas en educación infantil. Para la selección de estos partimos de las siguientes premisas: más de 20 años de labor profesional en el ámbito de la educación infantil, vinculados al ámbito universitario, con conocimiento de la literatura infantil y de la educación en valores. De estos, dos eran profesores de la Universidad de Granada, Doctores en Pedagogía, maestros de Educación Infantil, como primera titulación profesional. Y las otras tres eran profesoras de la Universidad de Córdoba, dos de ellas doctoras en Psicopedagogía, y la tercera doctora en Pedagogía. Las tres habían ejercido como docentes en la etapa de Educación Infantil durante 4,8 y 10 años respectivamente, siendo en la actualidad profesoras de universidad en el
Grado de Educación Infantil. Para la valoración de la escala se las entregamos para que aportaran aquello que consideraran oportuno; el equipo finalmente no incorporó ningún cuento ni tampoco ningún valor, dejando el instrumento tal cual lo habíamos diseñado.

La población participante en el estudio estaba conformada por los docentes que imparten sus clases en la etapa de Educación Infantil de la zona de Palma del Río en la provincia de Córdoba; constaba de 34 sujetos, de los cuales 26 conformaron la muestra objeto de estudio. En función de la variable de identificación sexo, todas ellas eran mujeres. Podría esto indicar la existencia de un sesgo en la población en lo que se refiere a la variable género, pero como señalan Gialamas, Nikiolopoulu y Koutromanos (2013), y Cheng y Chang (2006), los estudios de Educación Infantil y Primaria son carreras 
con un perfil eminentemente feminizado, afectando esta variable de manera mundial; es por ello que consideramos la no existencia de dicho sesgo en nuestra muestra, dado que la variable extraña que determina la asignación del alumnado a los grupos durante el proceso de matriculación está fuera del control de los investigadores e investigadoras, atendiendo las variables descriptivas -tipología del colegio, nivel educativo y años de experiencia-. En lo que se refiere a la tipología de los centros, debemos señalar que todos los sujetos participantes pertenecían a centros públicos, puesto que los de carácter privado o concertado que hay en la zona, no remitieron el cuestionario. Por otra parte, es significativo destacar que todas las participantes presentan la misma titulación de ingreso en el cuerpo de docentes (diplomatura en Maestro especialidad de Educación Infantil). En función de la muestra por edad, un 19,2\% tiene entre 36 a 40 años, seguidas por $11,5 \%$ entre 25 y 30 años, a continuación un 7,7\% entre 31 a 35 años, y por último un $3.8 \%$ son menores de 25 años. En función del nivel educativo, destaca la participación de profesoras del nivel educativo de 3 años, a continuación de 5 años, y por último del nivel educativo de 4 años. En cuanto a los años de experiencia, la muestra presenta un 53.8\% con más de 12 años de experiencia, seguido del $19,2 \%$ con 9 a 12 años, luego un $15,4 \%$ con 1 a 3 años y por último, un $11,5 \%$ con una experiencia de 4 a 8 años.

Gráfica 1. Distribución de la muestra en función de la edad.

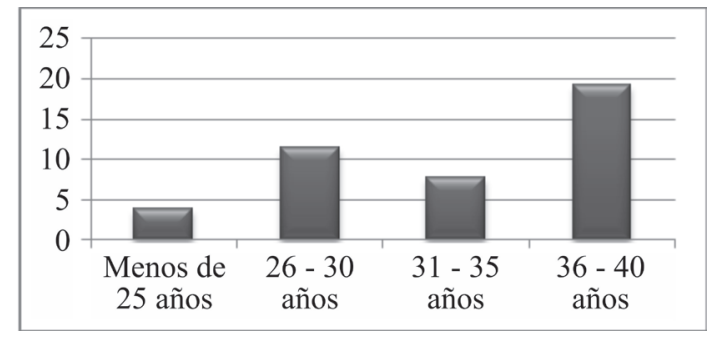

Fuente: Elaboración propia.

Para evaluar los datos recogidos a través de los diferentes instrumentos, empleamos el programa SPSS en su versión 20.0 para Windows, pues fue más fácil obtener, además de dejarnos realizar de forma más detallada el análisis de los datos cuantitativos, obtenidos después de haber aplicado la escala.

En cuanto al diseño de la investigación para el análisis de los resultados, hicimos un primer estudio de las variables de identificación para poder describir la muestra participante en el estudio y, dado el tipo de escala que empleamos -tabla de doble entrada- solo pudimos realizar un análisis descriptivo de los datos recogidos, el cual está compuesto por los estadísticos descriptivos básicos: media aritmética, desviación típica, distribución de frecuencias y porcentaje de cada una de las variables, los que nos van a ayudar a describir la muestra y nos darán una visión global de la misma, así como las contingencias en función de la edad y la experiencia profesional, desestimando la contingencia referida al sexo, dado que toda la muestra es fémina. Seguidamente realizamos las contingencias pertinentes referidas a la descripción de los valores en los cuentos estudiados; en tercer lugar, las señaladas en función del nivel educativo en que los profesores y profesoras llevan a cabo su labor, y por último las referidas a los años de experiencia.

\section{Resultados}

A partir de los datos obtenidos tras aplicar la escala de valoración, pudimos describir los valores que más incidencia tienen en los 19 cuentos tradicionales seleccionados a partir de la investigación llevada a cabo por Teres y García en 1997.

Atendiendo al objetivo uno de esta investigación -Determinar los valores que transmiten los cuentos infantiles seleccionadosy una vez recategorizados los porcentajes obtenidos, comprobamos que los valores que más presencia tienen en los cuentos son (ver tabla 3 y gráfica 1$)$ : ayuda $(39,67 \%)$, responsabilidad $(23,27 \%)$, justicia $(21,64 \%)$, cooperación $(22,56 \%)$ y respeto mutuo $(20,04 \%)$. En el otro extremo encontramos la paz $(6,06 \%)$ y la igualdad (6,46\%). El resto de valores tiene una presencia media en todos los cuentos. 
Tabla 3. Presencia de valores en los cuentos.

\begin{tabular}{|c|c|c|c|c|c|c|c|c|c|c|c|c|c|}
\hline & 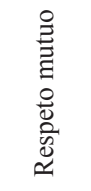 & 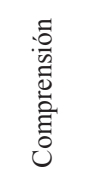 & 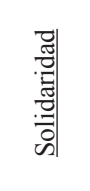 & 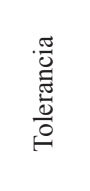 & 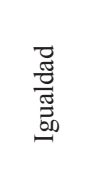 & 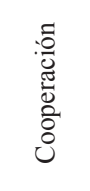 & 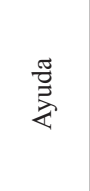 & 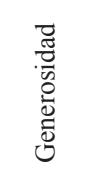 & 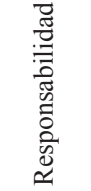 & है & 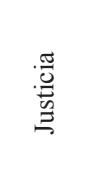 & 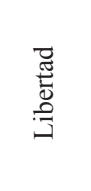 & $\frac{\vec{\pi}}{\frac{\pi}{0}}$ \\
\hline El patito feo & $53,8 \%$ & $23,1 \%$ & $19,2 \%$ & $50 \%$ & $23,1 \%$ & $0 \%$ & $26,9 \%$ & $7,7 \%$ & $0 \%$ & $0 \%$ & $7,7 \%$ & $3,8 \%$ & $7,7 \%$ \\
\hline Caperucita Roja & $3,8 \%$ & $15,4 \%$ & $0 \%$ & $0 \%$ & $0 \%$ & $0 \%$ & $76,9 \%$ & $26,9 \%$ & $42,3 \%$ & $0 \%$ & $11,5 \%$ & $0 \%$ & $11,5 \%$ \\
\hline El flautista de Hamelín & $0 \%$ & $0 \%$ & $7,7 \%$ & $3,8 \%$ & $0 \%$ & $26,9 \%$ & $53,8 \%$ & $0 \%$ & $34,6 \%$ & $7,7 \%$ & $76,9 \%$ & $11,5 \%$ & $19,2 \%$ \\
\hline Hansel y Gretel & $15,4 \%$ & $11,5 \%$ & $7,7 \%$ & $0 \%$ & $0 \%$ & $36,4 \%$ & $53,8 \%$ & $3,8 \%$ & $26,9 \%$ & $0 \%$ & $15,4 \%$ & $38,5 \%$ & $0 \%$ \\
\hline La Bella Durmiente & $3,8 \%$ & $7,7 \%$ & $11,5 \%$ & $11,5 \%$ & $0 \%$ & $7,7 \%$ & $42,3 \%$ & $11,5 \%$ & $3,8 \%$ & $7,7 \%$ & $19,2 \%$ & $0 \%$ & $7,7 \%$ \\
\hline El gato con botas & $3,8 \%$ & $7,7 \%$ & $26,9 \%$ & $11,5 \%$ & $3,8 \%$ & $46,2 \%$ & $30,8 \%$ & $38,5 \%$ & $26,9 \%$ & $0 \%$ & $11,5 \%$ & $0 \%$ & $11,5 \%$ \\
\hline Los tres cerditos & $15,4 \%$ & $7,7 \%$ & $38,5 \%$ & $3,8 \%$ & $0 \%$ & $42,3 \%$ & $38,5 \%$ & $7,7 \%$ & $42,3 \%$ & $0 \%$ & $26,9 \%$ & $7,7 \%$ & $0 \%$ \\
\hline Peter Pan & $15,4 \%$ & $15,4 \%$ & $34,6 \%$ & $3,8 \%$ & $19,2 \%$ & $26,9 \%$ & $19,2 \%$ & $15,4 \%$ & $7,7 \%$ & $7,7 \%$ & $42,3 \%$ & $38,5 \%$ & $0 \%$ \\
\hline La Cenicienta & $19,2 \%$ & $15,4 \%$ & $7,7 \%$ & $11,5 \%$ & $19,2 \%$ & $19,2 \%$ & $34,6 \%$ & $30,8 \%$ & $11,5 \%$ & $3,8 \%$ & $42,3 \%$ & $7,7 \%$ & $46,2 \%$ \\
\hline Los 7 cabritillos & $11,5 \%$ & $3,8 \%$ & $15,4 \%$ & $0 \%$ & $0 \%$ & $7,7 \%$ & $38,5 \%$ & $0 \%$ & $26,9 \%$ & $7,7 \%$ & $26,9 \%$ & $15,4 \%$ & $11,5 \%$ \\
\hline Los tres osos & $23,1 \%$ & $11,5 \%$ & $7,7 \%$ & $19,2 \%$ & $3,8 \%$ & $15,4 \%$ & $38,5 \%$ & $23,1 \%$ & $19,2 \%$ & $7,7 \%$ & $3,8 \%$ & $3,8 \%$ & $7,7 \%$ \\
\hline Pulgarcito & $11,5 \%$ & $3,8 \%$ & $3,8 \%$ & $3,8 \%$ & $0 \%$ & $26,9 \%$ & $57,7 \%$ & $15,4 \%$ & $23,1 \%$ & $3,8 \%$ & $3,8 \%$ & $11,5 \%$ & $0 \%$ \\
\hline Pinocho & $11,5 \%$ & $3,8 \%$ & $3,8 \%$ & $19,2 \%$ & $0 \%$ & $3,8 \%$ & $15,4 \%$ & $26,9 \%$ & $42,3 \%$ & $7,7 \%$ & $26,9 \%$ & $19,2 \%$ & $46,2 \%$ \\
\hline Blancanieves & $26,9 \%$ & $11,5 \%$ & $15,4 \%$ & $3,8 \%$ & $7,7 \%$ & $15,4 \%$ & $42,3 \%$ & $38,5 \%$ & $15,4 \%$ & $0 \%$ & $42,3 \%$ & $15,4 \%$ & $15,4 \%$ \\
\hline Bambi & $19,2 \%$ & $11,5 \%$ & $23,1 \%$ & $7,7 \%$ & $3,8 \%$ & $42,3 \%$ & $30,8 \%$ & $34,6 \%$ & $30,8 \%$ & $30,8 \%$ & $3,8 \%$ & $23,1 \%$ & $11,5 \%$ \\
\hline La lechera & $0 \%$ & $3,8 \%$ & $0 \%$ & $0 \%$ & $7,7 \%$ & $0 \%$ & $0 \%$ & $3,8 \%$ & $46,2 \%$ & $0 \%$ & $7,7 \%$ & $7,7 \%$ & $3,8 \%$ \\
\hline El mago de Oz & $42,3 \%$ & $23,1 \%$ & $19,2 \%$ & $26,9 \%$ & $3,8 \%$ & $46,2 \%$ & $34,6 \%$ & $7,7 \%$ & $3,8 \%$ & $7,7 \%$ & $7,7 \%$ & $30,8 \%$ & $3,8 \%$ \\
\hline La Bella y la Bestia & $65,4 \%$ & $69,2 \%$ & $26,9 \%$ & $42,3 \%$ & $15,4 \%$ & $15,4 \%$ & $42,3 \%$ & $46,2 \%$ & $15,4 \%$ & $11,5 \%$ & $15,4 \%$ & $30,8 \%$ & $19,2 \%$ \\
\hline El libro de la selva & $38,8 \%$ & $30,8 \%$ & $38,5 \%$ & $30,8 \%$ & $15,4 \%$ & $50 \%$ & $76,9 \%$ & $26,9 \%$ & $23,1 \%$ & $11,5 \%$ & $19,2 \%$ & $42,3 \%$ & $15,4 \%$ \\
\hline
\end{tabular}

Fuente: Elaboración propia.

Gráfica 2. Presencia de valores en los cuentos.

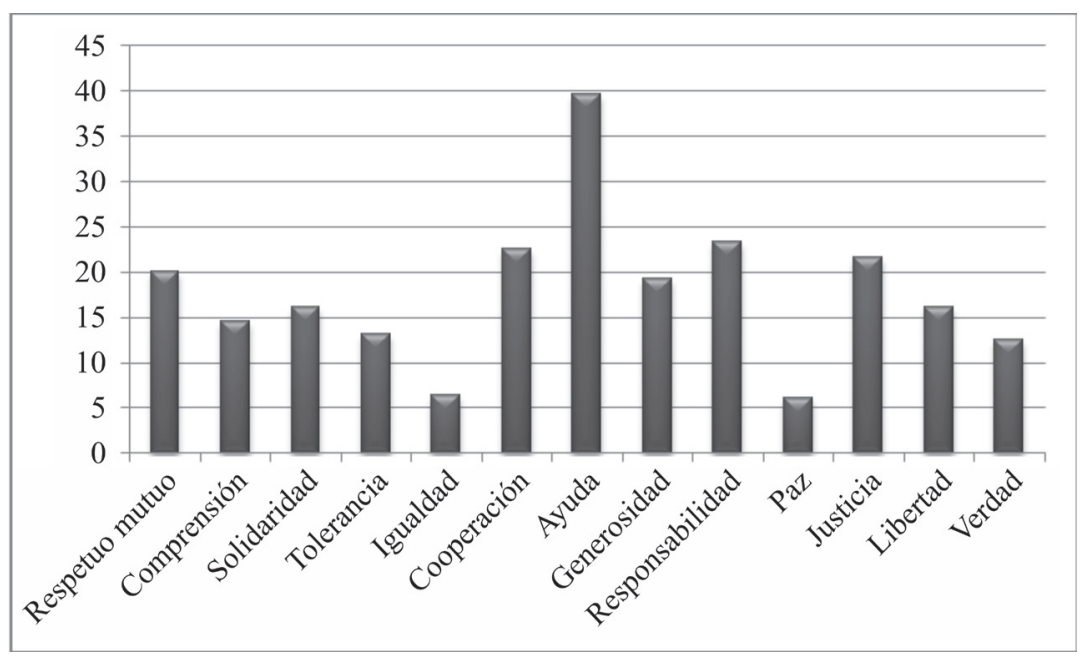

Fuente: Elaboración propia. 
En lo que se refiere al tercer objetivo -Determinar el número de valores que puede transmitir un cuento-, a partir de los datos de la tabla 3 comprobamos que de los 19 cuentos propuestos, 6 de ellos (La Cenicienta, Los Tres osos, Bambi, El mago de Oz, La Bella y la Bestia y El libro de la Selva) transmiten, a juicio de las docentes participantes, los 12 valores determinados. No siendo así en el caso de los cuentos "El flautista de Hamelin" y "La Lechera", que tan solo transmiten 7 valores, siendo la responsabilidad $(46,2 \%)$ y la justicia $(76,9 \%)$ los que más se potencian, respectivamente.

En cuanto a los resultados obtenidos con respecto al tercer objetivo -Determinar los valores que según los docentes adquirirán los estudiantes y las estudiantes en función del nivel educativo en que se encuentren-, los valores qué más se trabajan en los cuentos seleccionados en función del nivel educativo son los que se pueden apreciar en la tabla 4.

Tabla 4. Valores de los cuentos en función del nivel educativo.

\begin{tabular}{|c|c|c|c|c|}
\hline Cuento & $\mathbf{3}$ años & $\mathbf{4}$ años & $\mathbf{5}$ años & $\begin{array}{c}\text { Apoyo o } \\
\text { Refuerzo }\end{array}$ \\
\hline Patito feo & Tolerancia & Libertad & Justicia y Verdad & Generosidad \\
\hline Caperucita roja & Generosidad & Respeto mutuo & Cooperación & Justicia y verdad \\
\hline El flautista de Hamelin & Responsabilidad & Verdad & Paz & Ayuda \\
\hline Hansel y Gretel & Respeto mutuo & Comprensión & Generosidad & Responsabilidad \\
\hline La Bella Durmiente & Comprensión & Respeto mutuo & Libertad & Cooperación \\
\hline El gato con botas & Comprensión & Igualdad & Respeto mutuo & Verdad \\
\hline Los tres cerditos & Comprensión & Tolerancia & Respeto mutuo & Generosidad \\
\hline Peter Pan & Respeto mutuo & Solidaridad & Tolerancia & Paz \\
\hline La Cenicienta & Justicia & Solidaridad & Libertad & Paz \\
\hline Los siete cabritillos & Responsabilidad & Respeto mutuo & Comprensión & Paz \\
\hline Los tres osos & Comprensión & Paz y verdad & Igualdad & Justicia \\
\hline Pulgarcito & Ayuda y libertad & Justicia & Comprensión y & Ayuda \\
\hline Pinocho & Paz & Comprensión & Respeto mutuo & Cooperación \\
\hline Blancanieves & Generosidad & Libertad & Tolerancia & Solidaridad \\
\hline Bambi & Cooperación & Comprensión & Verdad & Justicia \\
\hline La Lechera & Justicia y libertad & Generosidad & Comprensión & Verdad \\
\hline El mago de Oz & Libertad & Justicia & Igualdad & Ayuda \\
\hline La Bella y la Bestia & Igualdad & Libertad & Libertad & Paz \\
\hline El libro de la selva & Verdad & Responsabilidad & Tolerancia & Comprensión \\
\hline
\end{tabular}

Fuente. Elaboración propia

Atendiendo al nivel educativo, podemos señalar que los cuentos que desarrollan más valores en el nivel de tres años son El patito feo, Los tres cerditos, Los tres osos, El libro de la selva y Blancanieves. Estos cuentos, por lo tanto, son los que más concuerdan con las características psicoevolutivas del alumnado de esta edad. En el nivel de cuatro años, los que se adecuan mejor al alumnado de estas edades son los siguientes: Caperucita Roja, La Bella durmiente, Blancanieves, Pinocho, La Bella y la Bestia. Por último, en cinco años los cuentos son El flautista de Hamelín, Hansel y Gretel, El gato con botas, Peter Pan, La Cenicienta, 
Los siete cabritillos, Pulgarcito y El mago de Oz. Son cuentos más complejos en cuanto a las características psicológicas de los personajes y en cuanto a las moralejas que nos transmiten.

Tabla 5. Valores de los cuentos en función de los años de experiencia.

\begin{tabular}{|c|c|c|c|c|}
\hline Cuento & $\mathbf{1}$ a 3 años & 4 a 8 años & 9 a 12 años & Más de 12 años \\
\hline Patito feo & Libertad & Comprensión & Comprensión & Verdad \\
\hline Caperucita roja & Respeto mutuo & Cooperación & Responsabilidad & Comprensión \\
\hline El flautista de Hamelin & Tolerancia & Paz & Paz & Respeto mutuo \\
\hline Hansel y Gretel & Responsabilidad & Comprensión & Comprensión & Solidaridad \\
\hline La Bella Durmiente & Respeto mutuo & Solidaridad & Comprensión & Responsabilidad \\
\hline El gato con botas & Igualdad & Comprensión & Justicia & Tolerancia \\
\hline Los tres cerditos & Generosidad & Comprensión & Comprensión & Respeto mutuo \\
\hline Peter Pan & Respeto mutuo & Paz & Comprensión & Solidaridad \\
\hline La Cenicienta & Solidaridad & Paz & Tolerancia & Responsabilidad \\
\hline Los siete cabritillos & Paz & Comprensión & Solidaridad & Respeto mutuo \\
\hline Los tres osos & Justicia & Igualdad & Generosidad & Respeto mutuo \\
\hline Pulgarcito & Respeto mutuo & Cooperación & Comprensión & Solidaridad \\
\hline Pinocho & Comprensión & Respeto mutuo & Paz & Solidaridad \\
\hline Blancanieves & Igualdad & Comprensión & Ayuda & Tolerancia \\
\hline Bambi & Justicia & Libertad & Libertad & Comprensión \\
\hline La Lechera & Generosidad & Verdad & Respeto mutuo & Igualdad \\
\hline El mago de Oz & Paz & Cooperación & Cooperación & Generosidad \\
\hline La Bella y la Bestia & Paz & Libertad & Paz & Responsabilidad \\
\hline El libro de la selva & Comprensión & Paz & Igualdad & Solidaridad \\
\hline
\end{tabular}

Fuente: Elaboración propia

Como podemos observar en la tabla 5, el valor que más se destaca en todos los años de experiencia es lacomprensión; en el caso extremo encontramos la cooperación. Atendiendo a cada intervalo de años de experiencia, según las docentes con 1 a 3 años de labor profesional el valor que más se desarrolla es el respeto mutuo, frente a las que llevan entre 4 y 12 años de trabajo como profesoras quienes consideran que es la comprensión, como apuntábamos antes. Por último, las docentes que podríamos catalogar de veteranas o "expertise" opinan que el respeto mutuo junto con la responsabilidad son los valores determinantes del proceso educativo a través de los cuentos tradicionales.

\section{Conclusiones}

Trabajar en la etapa de educación infantil supone hoy un gran reto, fundamentalmente derivado de la gran heterogeneidad que las aulas presentan. Es a partir de esta que los profesores y profesoras deben plantear metodologías de aula que sustenten una formación que los niños y niñas puedan implementar de manera correcta en los niveles superiores de la educación. En esta línea, la educación en valores ha sido tratada de forma coyuntural y transversal a todas las materias del currículo.

Los datos encontrados en este estudio han revelado que las hipótesis de partida se confirman, en el sentido de que, 
independientemente de los años de experiencia profesional que los sujetos docentes puedan presentar, los cuentos tradicionales transmiten valores, y estos pueden ser considerados como elementos clave en la construcción del yo del individuo (Eming \& Fujimoto-Gómez, 2003). Es significativo constatar que un cuento pueda transmitir más de un valor; sin embargo, es llamativo que el mismo cuento en función del nivel educativo así como de la experiencia profesional del profesorado, varíe significativamente, con lo cual inferimos que, o bien el proceso de socialización de los niños y niñas, o bien el bagaje profesional, determinan las percepciones de estos valores.

Por otra parte, nuestra segunda hipótesis de trabajo -la consideración de que los cuentos pueden ser empleados como elemento reforzador de un valor una vez se ha adquiridono se confirma, por lo que debemos rechazar así la idea de que los cuentos se puedan emplear como reforzadores de valores adquiridos previamente en cursos anteriores. Por ejemplo, al igual que sucede en la investigación de Andonovska-Trajkovska (2012), la edad de los niños y niñas influye en los valores que los cuentos transmiten; así, el cuento "La Bella Durmiente" en primero de Educación Infantil, potencia el valor de la comprensión; en segundo curso el respeto mutuo, y en último curso la libertad, incluida el aula de apoyo o de refuerzo en donde se trabaja la cooperación. No obstante, la hipótesis tercera -ayudan al docente en el desarrollo de la educación en valores- se refuerza en función de los años de experiencia profesional que los profesores y profesoras presentan, por lo que se puede aceptar esta posición.

En definitiva, consideramos que la formación en la etapa de Educación Infantil tiene un gran sustento en la formación en valores y en el proceso de construcción de la persona; es por ello que los cuentos tradicionales, tan presentes en este momento formativo, cobran una gran importancia ante el desarrollo de los valores que posteriormente guiará la vida de los individuos.

\section{Lista de referencias}

Amei-Waece -Asociación Mundial de Educadores Infantiles- (2010). El trabajo en valores con niños y niñas de 0 a 6 años. Revista Latinoamericana de Ciencias Sociales, Niñez y Juventud, 8 (1), pp. 683703.

Andonovska-Trajkosvska, D. (2012). Propp's functions recognized in the children's perceptions of the fairy tales. Procedia Social and Behavioral Sciences, 46, pp. 1695-1700.

Bassedas, E., Huguet, T., \& Solé, I. (1998). Aprender y enseñar en educación infantil. Barcelona: Graó.

Bruder, M. (2000). El cuento y los afectos. Buenos Aires: Galerna.

Cortes Generales de España (2013). Ley Orgánica para la Mejora de la Calidad Educativa. Madrid: BOE. Recuperado el 3 de febrero de 2014, de:

http://www.boe.es/boe/dias/2013/12/10/ pdfs/BOE-A-2013-12886.pdf

Cheng, J. \& Chang, C. (2006). Using computers in early childhood classrooms: teachers' attiudes, skills and practices. Journal of Early Childhood Research, 4 (2), pp. 269288.

Duch, P. (2003). Cuentos para amar los libros. Valencia: Brosquil, D. L.

Echevarría, C. V. (2003). La escuela un escenario de formación y socialización para la construcción de identidad moral. Revista Latinoamericana de Ciencias Sociales, Niñez y Juventud, 1 (2), pp. 1-26.

Eming, M. \& Fujimoto-Gómez, G. (2003). Desarrollo infantil temprano: lecciones de los programas no formales. Revista Latinoamericana de Ciencias Sociales, Niñez y Juventud, 1 (1), pp. 509-521.

García, G. E. \& Gallego, T. (2011). Una concepción abierta e interdisciplinar de la infancia. Revista Latinoamericana de Ciencias Sociales, Niñez y Juventud, 9 (2), pp. 17-25.

Gialamas, V., Nikolopouiou, K. \& Kutromanos, G. (2013). Student teachers' perceptions about the impact of internet usage on their 
learning and jobs. Computers \& Education, 62, pp. 1-7.

Doi:http://dx.doi.org/10.1016/j. compedu.2012.10.012.

González, I. (2006). El valor de los cuentos infantiles como recurso para trabajar la transversalidad en las aulas. Campo Abierto, 25 (1), pp. 11-29.

López, F. (2005). Desarrollo afectivo y social. Madrid: Pirámide.

Marín-Díaz, V., Llorent, V., González, M. \& Mata, J. M. (2005). La formación del profesorado universitario en educación en valores. Lusíada de Historia, II (2), pp. 325-336.

Martín, A. (2006). Vamos a contar un cuento. Revista Digital de Investigación y Educación, 22. Recuperado el 3 de febrero de 2014, de:

http://www.csi-csif.es/andalucia/modules/ mod_revistaense/archivos/N_22_2006/CUENTO_infan.pdf (03/02/2014).

Medina, A. (1973). Enciclopedia de didáctica aplicada. Barcelona: Labor.

Ortega, R. \& Tenorio, J. M. (2006). El cuento. Revista Digital de Investigación $y$ Educación, 26. Recuperado el 3 de febrero de 2014, de:

http://www.csi-csif.es/andalucia/modules/ mod_revistaense/archivos/N_26_2006/CUENTO_infan.pdf

Paya, M. (1997). Educación en valores para una sociedad abierta y plural: aproximación conceptual. Bilbao: Descleé De Brouwer.

Pestaña, P. (2004). Aproximación conceptual al mundo de los valores. Reice, Revista Electrónica Iberoamericana sobre Calidad, Eficacia y Cambio en Educación, 2 (2), pp. 67-82. Recuperado el 12 de junio de 2014, de:

http://www.rieoei.org/oeivirt/rie07a04.pdf

Poma, M. I. (2011). Programa en educación de valores para fortalecer la formación ciudadana y la cultura de paz en los niños de segundo año de Educación Básica Paralelo " $C$ " en la escuela Cristóbal Colón ubicada en el Cantón Salcedo provincia de Cotopaxi. Tesis Doctoral inédita, Universidad Técnica de Cotopaxi,
Latacunga, Ecuador. Recuperado el 12 de junio de 2014, de:

http://repositorio.utc.edu.ec/ bitstream/27000/1469/1/T-UTC-1345.pdf

Quintana, J. Ma (2005). La educación en valores y otras cuestiones pedagógicas. Barcelona: PPU.

Riviere, A. (1984). La psicología de Vigotsky. Madrid: Visor.

Rollano, D. (2004). Educación en valores. Vigo: Ideaspropias.

Stufflebeam, D. L. \& Shinkfield, A. J. (1990). Evaluación sistemática: teórica y práctica. Barcelona: Paidós.

Teres, M. D. \& García, F. (1997). Desarrollos curriculares para la educación infantil. Madrid: Escuela Española, S. A.

Trilla, J. (1995). Educación y valores controvertidos. Elementos para un planteamiento normativo sobre la neutralidad en las instituciones educativas. Revista Iberoamericana de Educación, 7, pp. 93-120. Recuperado el 3 de febrero de 2014, de: http://www.rieoei.org/oeivirt/ rie07a04.pdf

Vigotsky, L. S. (1984). Aprendizaje y desarrollo intelectual en la edad escolar. Infancia $y$ Aprendizaje, 27-28, pp. 105-116. 